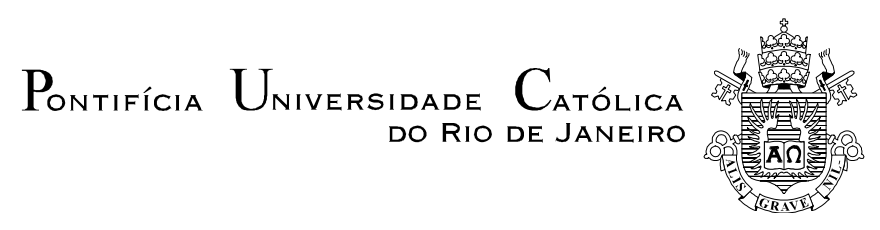

Priscila Marques Dias

\title{
Sistema de Contagem de Pessoas
}

\begin{abstract}
Dissertação de Mestrado
Dissertação apresentada como requisito parcial para obtenção do título de Mestre pelo Programa de PósGraduação em Engenharia Elétrica da PUC-Rio.

Orientador: Raul Queiroz Feitosa
\end{abstract}

Rio de Janeiro, abril de 2005. 


\title{
Priscila Marques Dias
}

\section{Sistema de Contagem de Pessoas}

\begin{abstract}
Dissertação apresentada como requisito parcial para obtenção do grau de Mestre pelo Programa de PósGraduação em Engenharia Elétrica do Departamento de Engenharia Elétrica do Centro Técnico Científico da PUCRio. Aprovada pela Comissão Examinadora abaixo assinada.
\end{abstract}

Prof. Raul Queiroz Feitosa

Orientador

Departamento de Engenharia Elétrica - PUC-Rio

Prof. Bruno Feijó

Departamento de Informática - PUC-Rio

Prof. Sidnei Paciornik

Departamento de Ciência dos Materiais e Metalurgia - PUC-Rio

Profa. Maria Luiza Fernandes Velloso

UERJ

Prof. José Eugenio Leal

Coordenador Setorial do Centro

Técnico Científico - PUC-Rio

Rio de Janeiro, 13 de abril de 2005. 
Todos os direitos reservados. É proibida a reprodução total ou parcial do trabalho sem autorização da universidade, da autora e do orientador.

\section{Priscila Marques Dias}

Graduou-se em Engenharia Elétrica com ênfase em Eletrônica no CEFET-RJ (Centro Federal de Educação Tecnológica Celso Suckow da Fonseca) em 2002.

Ficha Catalográfica

Dias, Priscila Marques

Sistema de contagem de pessoas / Priscila Marques Dias ; orientador: Raul Queiroz Feitosa. - Rio de Janeiro : PUC, Departamento de Engenharia Elétrica, 2005.

87 f. ; $30 \mathrm{~cm}$

Dissertação (mestrado) - Pontifícia Universidade Católica do Rio de Janeiro, Departamento de Engenharia Elétrica.

Inclui referências bibliográficas.

1. Engenharia elétrica - Teses. 2. Visão computacional. 3. Sistemas de segurança e supervisão. 4. Contagem de pessoas. 5. Detecção de atitudes suspeitas. I. Feitosa, Raul Queiroz. II. Pontifícia Universidade Católica do Rio de Janeiro. Departamento de Engenharia Elétrica. III. Título. 


\section{Agradecimentos}

Ao orientador Raul Queiroz Feitosa pela paciência, estímulo e confiança dispensados para a realização deste trabalho.

Ao CNpq e à PUC-Rio, pelos auxílios concedidos, sem os quais este trabalho não poderia ter sido realizado.

Aos professores que participaram da comissão examinadora.

Aos meus pais, Ernani e Celeste, à minha irmã e ao meu cunhado, Cristina e Ricardo, e ao meu marido, Leonardo.

Às amigas do Departamento de Engenharia Elétrica que muito me incentivaram e ajudaram: Karla Figueiredo e Marley Vellasco.

A todos os amigos e familiares que de uma forma ou de outra me estimularam e apoiaram.

A Deus. 


\section{Sumário}

1 Introdução

2 Abordagens Anteriores $\quad 19$

2.1. Separação do Fundo / Primeiro Plano 20

2.2. Atualização da Estimativa do Fundo 23

2.3. Localização / Contagem de Pessoas 25

3 Solução Proposta 32

3.1. Captura e Pré-Processamento da Imagem 32

3.2. Separação do Fundo / Primeiro Plano 33

3.2.1. Tratamento de Sombras $\quad 34$

3.2.2. Tratamento de Ruído 36

3.3. Atualização da Estimativa do Fundo 37

3.4. Localização / Contagem de Pessoas 39

3.4.1. Segmentação do Primeiro Plano 39

3.4.2. Agrupamento das Regiões de Interesse 42

3.5. Detecção de Atitudes Suspeitas $\quad 47$

4 Avaliação Experimental $\quad 48$

4.1. Descrição dos Ambientes 48

4.2. Experimentos com Seqüências de Vídeo de Situações Simuladas 49

4.2.1. Separação do Fundo / Primeiro Plano 50

4.2.2. Atualização da Estimativa do Fundo 53

4.2.3. Localização / Contagem de Pessoas $\quad 59$

4.2.4. Detecção de Atitudes Suspeitas 69

4.3. Experimentos com Seqüências de Vídeo de Situações Reais 70

4.4. Sensibilidade aos Valores dos Parâmetros 73

$\begin{array}{ll}5 \text { Comentários Finais } & 76\end{array}$

$\begin{array}{ll}\text { Referências Bibliográficas } & 79\end{array}$ 
Apêndice A

Apêndice B

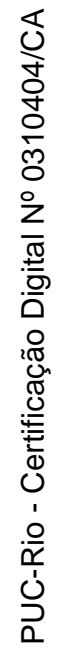




\section{Lista de figuras}

Figura 1: Estrutura básica do sistema proposto. $\quad 19$

Figura 2: Sistema $W^{4}$ de Haritaoglu. $\quad 27$

Figura 3: Algoritmo convencional de separação do fundo / primeiro plano. $\quad 34$

Figura 4: Algoritmo de separação do fundo / primeiro plano com tratamento de sombras. $\quad 35$

Figura 5: Resultados do algoritmo de separação do fundo / primeiro plano: (a) imagem de entrada; (b) pixels relativos ao fundo; (c) pixels relativos ao fundo e a sombras; (d) pixels relativos ao fundo e a sombras após operações morfológicas. $\quad 36$

Figura 6: Algoritmo de atualização da estimativa do fundo. 38

Figura 7: Processamento da imagem antes de ser submetida ao algoritmo básico de watershed: (a) imagem de entrada; (b) após suavização; (c) média do gradiente nas três componentes de cores em 2D (d) e em 3D; (e) após superposição da grade em 2D (e) e em 3D (f); após supressão de mínimos em 2D (g) e em 3D (h). $\quad 41$

Figura 8: Resultado produzido pela segmentação do primeiro plano. 42

Figura 9: Correspondência das regiões de interesse. 43

Figura 10: Algoritmo para agrupamento de segmentos. 46

Figura 11: Resultado da agregação dos segmentos da Figura 8.

Figura 12: Seqüências para análise de sombras: (a) ambiente 1 sem ninguém; (b) ambiente 2 sem ninguém; (c) e (d) pessoa entra com objeto; (e) e (f) pessoa deixa objeto no local; (g) e (h) pessoa sai. 51

Figura 13: Sombras geradas: ambiente 1 - (a) suave; (b) média; (c) forte; ambiente 2 - (d) suave; (e) média; (f) forte. 52

Figura 14: Seqüência para análise da atualização do fundo nos casos de presença de pessoas: (a) ambiente 3 sem ninguém; (b) primeiro plano correspondente; (c) pessoa entra; (d) primeiro plano correspondente; (e) pessoa circula; (f) primeiro plano correspondente; (g) pessoa sai; (h) primeiro plano correspondente. 55

Figura 15: Condições de iluminação geradas: máxima - ambientes 1 (a), 2 (b) e 3 (c); mínima - ambientes 1 (d), 2 (e) e 3 (f).

Figura 16: Variação de iluminação gerada: (a) mínima; (b) máxima; (c) máxima; e respectiva variação das atualizações de fundo: (a) mínima; (b) mediana; (c) 
máxima.

Figura 17: Seqüência para análise da atualização do fundo nos casos de alterações permanentes: (a) ambiente 4 sem ninguém; (b) pessoa entra e pega um objeto; (c) pessoa se retira.

Figura 18: Atualização da estimativa de fundo relativa a alteração permanente da Figura 17: (a) lixeira pertence à estimativa; (b), (c), (d) e (e) lixeira aos poucos vai sendo eliminada da estimativa; (f) lixeira é totalmente eliminada da estimativa.

Figura 19: Seqüências para análise da atualização do fundo nos casos de alterações permanentes: ambientes 1 (a), 2 (b) e 3 (c) sem ninguém; pessoa entra e pega (d) ou deixa (e) (f) um objeto; pessoa se retira (g) (h) (i).

Figura 20: Seqüências para análise da localização / contagem de pessoas nos casos de oclusões: ambiente 1 (a), 2 (b) e 3 (c) sem ninguém; pessoa entra e circula (d) (e) (f); pessoa sofre oclusão (g) (h) (i); pessoa se retira (j) (l) (m).

Figura 21: Seqüência para análise da localização / contagem de pessoas nos casos de pessoas isoladas: (a) ambiente 2 sem ninguém; (b) pessoas entram em cena isoladamente e circulam pelo ambiente; (c) pessoas saem sem se tocar.

Figura 22: Seqüências para análise da localização / contagem de pessoas nos casos de formação de grupos: ambiente 1 (a), 2 (b) e 3 (c) sem ninguém; 2 (d), 3 (e) ou 4 (f) pessoas entram isoladamente na cena; grupos de 2 (g), 3 (h) ou 4 (i) pessoas se formam; grupos de 2 (j), 3 (l) ou 4 (m) pessoas se retiram.

Figura 23: Seqüências para análise da localização / contagem de pessoas nos casos de separação de grupos: ambiente 1 (a), 2 (b) e 3 (c) sem ninguém; grupos de 2 (d), 3 (e) ou 4 (f) pessoas entram na cena; grupos de 2 (g), 3 (h) ou 4 (i) pessoas se separam; 2 (j), 3 (l) ou 4 (m) pessoas saem isoladamente.

Figura 24: Seqüências para análise da detecção de atitudes suspeitas: ambiente 1 (a), 2 (b) e 3 (c) sem ninguém; pessoa entra e deixa (d) ou pega (e) (f) um objeto; pessoa se retira (g) (h) (i).

Figura 25: Seqüência para análise de uma situação real: (a) 2 adultos estão presentes na cena; (b) uma criança entra; (c) um outro adulto entra; (d) um adulto sai; (e) outro adulto sai; (f) a criança passa por trás do último adulto 
em cena e (g) segue para o outro lado da banca; ( $h$ ) o adulto também segue para o outro lado da banca; (i) o adulto se agacha e encobre a criança.

71

Figura 26: Conversão de RGB para CIE XYZ.

86

Figura 27: Conversão de CIE XYZ para CIE Lab. 


\section{Lista de tabelas}

Tabela 1: Descrição dos ambientes. $\quad 49$

Tabela 2: Percentual de sombra detectado. 52

Tabela 3: Relação entre o número de pessoas e a quantidade de quadros das seqüências de teste de atualização do fundo nos casos de presença de pessoas.

Tabela 4: Relação entre o número de pessoas e a quantidade de quadros das seqüências de teste de localização / contagem de pessoas nos casos de oclusões.

Tabela 5: Erro médio e desvio padrão encontrados nas seqüências de teste de localização / contagem de pessoas nos casos de oclusões.

Tabela 6: Relação entre o número de pessoas e a quantidade de quadros das seqüências de teste de localização / contagem de pessoas nos casos de pessoas isoladas.

Tabela 7: Relação entre o número de pessoas e a quantidade de quadros das seqüências de teste de localização / contagem de pessoas nos casos de formação de grupos.

Tabela 8: Relação entre o número de pessoas e a quantidade de quadros das seqüências de teste de localização / contagem de pessoas nos casos de separação de grupos.

Tabela 9: Erro médio e desvio padrão encontrados nas seqüências de teste de localização / contagem de pessoas nos casos de pessoas isoladas circulando.

Tabela 10: Erro médio e desvio padrão encontrados nas seqüências de teste de localização / contagem de pessoas nos casos de formação de grupos de 2 , 3 e 4 pessoas.

Tabela 11: Erro médio e desvio padrão encontrados nas seqüências de teste de localização / contagem de pessoas nos casos de separação de grupos de 2, 3 e 4 pessoas.

Tabela 12: Erro médio e desvio padrão de acordo com o número de pessoas em cena.

Tabela 13: Relação entre o número de pessoas e a quantidade de quadros da seqüência de teste de uma situação real.

Tabela 14: Erro médio e desvio padrão encontrados na seqüência de teste de 
uma situação real.

72

Tabela 15: Descrição e valores dos parâmetros fixos do sistema. 73

Tabela 16: Descrição e valores dos parâmetros ajustáveis do sistema. $\quad 74$

Tabela 17: Símbolos utilizados no texto e suas respectivas descrições. 84 


\section{Resumo}

Atualmente, a preocupação com segurança vem crescendo dia após dia. Vários trabalhos abordando o desenvolvimento de sistemas de supervisão já foram realizados. Esta dissertação propõe um método automático capaz de determinar o número de pessoas em uma área monitorada por uma câmera de vídeo, assim como detectar mudanças na imagem potencialmente causadas por atitudes ilícitas. Uma aplicação típica seria a segurança de galpões durante a noite, em finais de semana ou em qualquer momento onde 0 acesso de pessoas é permitido, mas o movimento de cargas não. Mais precisamente, a intenção é detectar se uma pessoa que está passando pelo ambiente carrega consigo um objeto pertencente ao local ou deixa um objeto no local, quando apenas o movimento de pessoas é admitido na área. Além disto, o sistema determina o número de pessoas na cena. O método consiste na aplicação de quatro etapas em seqüências de vídeo: a) separação de fundo / primeiro plano, b) atualização dinâmica da estimativa de fundo, c) localização / contagem de pessoas, e d) detecção de atitudes suspeitas. Os algoritmos de separação de fundo / primeiro plano e de estimativa de fundo toleram variações pequenas de iluminação e efeitos de sombra. Já a contagem / localização de pessoas explora informações de cor e coerência de movimento. Soluções para atender estes aspectos são encontradas na literatura, porém nenhuma delas atende todos eles juntos. $O$ método foi avaliado por experimentos realizados através de um protótipo e apresentou resultados encorajadores.

\section{Palavras-chave}

Visão Computacional, Sistemas de Segurança e Supervisão, Contagem de Pessoas, Detecção de Atitudes Suspeitas. 


\section{Abstract}

There is worldwide an increasing concern about security issues. A great deal of efforts have been undertaken in order to provide surveillance systems. This work proposes an automatic method to determine the number of people moving in an area monitored by a video camera, as well as to detect image changes, which are potentially due to illicit attitudes. A typical application is the security of warehouses during the night, on weekends or at any time when people access is allowed but no load movement is admissible. Specifically it focuses on detecting when a person passing by the environment carries any object belonging to the background away or leaves any object in the background, while only people movement is allowed in the area. Besides it estimates the number of people on scene. The method consists of performing four main tasks on video sequences: a) background and foreground separation, b) background estimative dynamic update, c) people location and counting, and d) suspicious attitudes detection. The proposed background and foreground separation and background estimative update algorithms deal with illumination fluctuation and shade effects. People location and counting explores colour information and motion coherence. Solutions meeting these requirements are proposed in the literature, but no one deals with all of them together. The method has been validated by experiments carried out on a prototype and produced encouraging results.

\section{Keywords}

Computer Vision, Security and Surveillance Systems, People Counting, Suspicious Attitudes Detection. 